\title{
Fluctuational Escape from Chaotic Attractors
}

\author{
I. A. Khovanov*, D. G. Luchinsky ${ }^{\dagger}$, R. Mannella**, P. V. E. McClintock ${ }^{\dagger}$ \\ and A. N. Silchenko \\ ${ }^{*}$ Department of Physics, Saratov State University, Astrahanskaya 83, 410026, Saratov, Russia \\ ${ }^{\dagger}$ Department of Physics, Lancaster University, Lancaster, LA1 4YB, UK \\ ** Dipartimento di Fisica, Università di Pisa and INFM UdR Pisa, 56100 Pisa, Italy
}

\begin{abstract}
Fluctuational transitions between two coexisting attractors are investigated. Two different systems are considered: the periodically driven nonlinear oscillator and the two-dimensional map introduced by Holmes. These two systems have smooth and fractal boundaries, respectively, separating their coexisting attractors. It is shown that, starting from a cycle embedded in the chaotic attractor, the periodically-driven oscillator escapes to a saddle cycle at the boundary of the basin of attraction, and does so through sequential transitions between saddles cycles embedded in the attractor. In the case of discrete dynamics with locally disconnected fractal boundaries, it is shown that escape from an attractor always seems to occur through an accessible boundary orbit and further through the specific homoclinic points forming a fractal structure of the boundary. It is shown that analysis of fluctuational transitions between attractors can be used to solve a problem of the energy-optimal migration of a chaotic system. The deterministic optimal control functions are identified with the corresponding optimal fluctuational forces in the limit of small noise intensity. We discuss possible applications and related unsolved problems of stochastic dynamics.
\end{abstract}

\section{INTRODUCTION}

The way in which fluctuational escape occurs from a chaotic attractor (CA) subject to noise has been a major unsolved problem ever since the first attempt to generalize the classical escape problem to cover this case [1]. We have recently used measurements of the prehistory probability distribution to analyse [2] escape from a chaotic attractor to a coexisting stable limit cycle in a periodically driven nonlinear oscillator - a situation where the basin boundaries are smooth. In the present work, we summarise these results, set them in context, and describe a completely new study in which we have extended the investigation to treat the discrete dynamics of a 2-dimensional map [3] for which the basin boundaries are fractal.

As in our earlier study [2], we bring together two seemingly quite different unsolved problems: first, that of fluctuational escape from a chaotic attractor and, secondly, the energy-optimal stimulation of escape in the absence of noise. We show that, remarkably, a solution of the first problem leads naturally to a solution of the second one.

The methods already available [4] for the control of chaos include entraining to a chosen "goal dynamics" that necessarily requires large modifications of the system's dynamics, and a variety of minimal forms of interaction which have hitherto been restricted by the linear approximations adopted. Solution of the energy-optimal control problem constitutes an important extension of the range of model-exploration objectives achievable through minimal control techniques. 
The main difficulty in tackling these problems stems from the complexity of the system dynamics near a CA and in general near boundaries, which may be fractal. The problems are related, in particular, to delicate questions concerning the uniqueness of the solution and the boundary conditions at a CA with fractal basin boundaries. The approach that we apply is based on the analysis of an oscillator interacting with a thermal bath. In the zero-noise-intensity limit, a consistent theoretical development [5, 6] from the microscopic to the macroscopic equations of motion leads to descriptions of both its deterministic (dissipative) and fluctuational dynamics within the framework of Hamiltonian formalism [7]. It can be shown on physical grounds, and rigorously, that the Wentzel-Freidlin Hamiltonian [7] arising in this approach is equivalent to the Pontryagin Hamiltonian in the control problem [8] with an additive linear unrestricted control; the corresponding optimal control function is equivalent to the optimal fluctuational force. The solution of the energy-optimal control problem can thus be found to an excellent approximation by building the prehistory probability distribution [9] of fluctuational escape trajectories.

We illustrate the approach by analysing and comparing two very different cases: escape from the chaotic attractor of a periodically driven nonlinear oscillator where the basin boundaries are smooth [2]; and escape from the chaotic attractor of an invertible 2-dimensional map with fractal basin boundaries [3].

\section{CONTINUOUS-TIME DYNAMICS}

We consider a periodically driven nonlinear oscillator of form

$$
\begin{aligned}
\dot{q}_{1}= & q_{2}, \\
\dot{q}_{2}= & -2 \Gamma q_{2}-\omega_{0}^{2} q_{1}-\beta q_{1}^{2}-\gamma q_{1}^{3}-h \cos (\Omega t)+\xi(t), \\
& \langle\xi(t)\rangle=0, \quad\langle\xi(t) \xi(0)\rangle=D \delta(t) .
\end{aligned}
$$

Here $\xi(t)$ is the Gaussian white noise. Parameters were chosen such that the potential is monostable $\left(\beta^{2}<4 \gamma \omega_{0}^{2}\right)$, the dependence of the energy of oscillations on their frequency is nonmonotonic $\left(\beta^{2} / \gamma \omega_{0}^{2}>9 / 10\right)$, and the motion is underdamped $\Gamma \ll \Omega \approx 2 \omega_{0}$. This model is of interest in number of applications and allows a theoretical analysis to be carried out over a wide range of parameters values. It is a system in which chaos can conveniently be observed at relatively small values $h \approx 0.1$ of the driving force amplitude.

For the given parameters $\omega_{0} \approx 0.597, \beta=1, \gamma=1$ and, with damping constant $\Gamma=0.025$, the amplitude and frequency of the driving force were chosen such that the chaotic attractor coexists with the stable limit cycle. The chaotic state appears as the result of period-doubling bifurcations and thus corresponds to a nonhyperbolic attractor (NHA). The boundary of attraction $\partial \Omega$ of the NHA is non-fractal and is formed by the stable manifold of the saddle cycle of period 1.

Direct application of Hamiltonian formalism [7] consists of consideration of a boundary problem and requires the identification of the generally unknown boundary conditions on the CA and on the boundaries of attraction (for fractal boundaries) and relies 


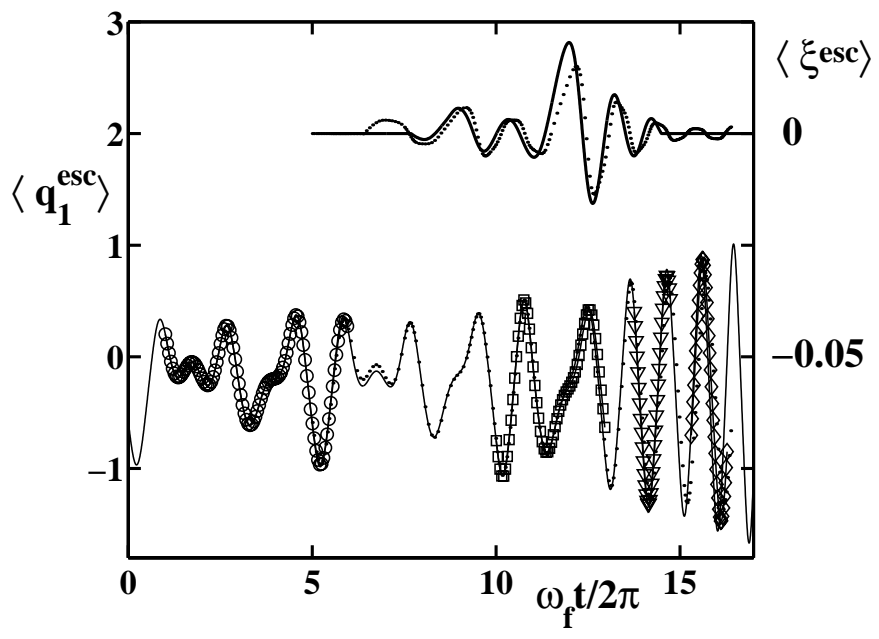

FIGURE 1. The most probable escape path (bottom, full curve) from the NHA to the boundary saddle cycle of period 1 , found in numerical simulations with $\Gamma=0.025, h=0.13, \Omega=0.95, D \approx 0.01$. Single periods of the unstable saddle cycles of period 5, 3 and 1 are shown by open circles, squares and triangles respectively; the stable limit cycle is shown by rhombus. The corresponding optimal force (top) after filtration. The optimal path and the optimal fluctuational force found by solution of the boundary value problem for motion equations with Wentzel-Freidlin Hamiltonian, corresponding to the system 1, are shown as dotted lines. After [2].

on the existence of the so-called most probable escape (optimal) path and corresponding optimal fluctuational force, which coincides with the optimal control function in the control problem. The use of the experimental prehistory technique [9] can solve all these problems and gives accurate approximation for the optimal control force.

The idea underlying our experimental technique is that, when the system (1) is driven by a random force $\xi(t)$ it will occasionally fluctuate to $\partial \Omega$. In doing so in the limit where the noise intensity tends to zero, the system will follow very closely the optimal escape path.

The method involves following the dynamics of the system continuously and collecting all successful realizations $\left(q_{1}^{\text {esc }}(t), q_{2}^{\text {esc }}(t), \xi^{\text {esc }}(t)\right)$ that move it from the NHA to $\partial \Omega$. An approximate control function is then found as an ensemble average over the corresponding realizations of the random force $\left\langle\xi^{\mathrm{esc}}(t)\right\rangle$. The boundary conditions are found from an analysis of how the energy-optimal escape path $\left(\left\langle q_{1}^{\text {esc }}(t)\right\rangle,\left\langle q_{2}^{\text {esc }}(t)\right\rangle\right)$ merges with the NHA.

A typical optimal escape path and the corresponding optimal force, obtained [2] by averaging a few hundred escape trajectories, are shown in Fig. 1. Analysis of the optimal path reveals that the system leaves the NHA along the unstable manifold of the saddle limit cycle of period 5 (with multipliers $\mu_{1}=0.04157<1$ and $\mu_{2}=4.60403728>1$ ) embedded in the NHA. At this moment the optimal fluctuational force $\left\langle\xi^{\operatorname{esc}}(t)\right\rangle$ switches on, driving the system to $\partial \Omega$ via the saddle cycle of period 3 (with multipliers $\mu_{1}=$ $0.04873<1$ and $\mu_{2}=7.608312>1$ ). Near the saddle cycle that forms the boundary of 

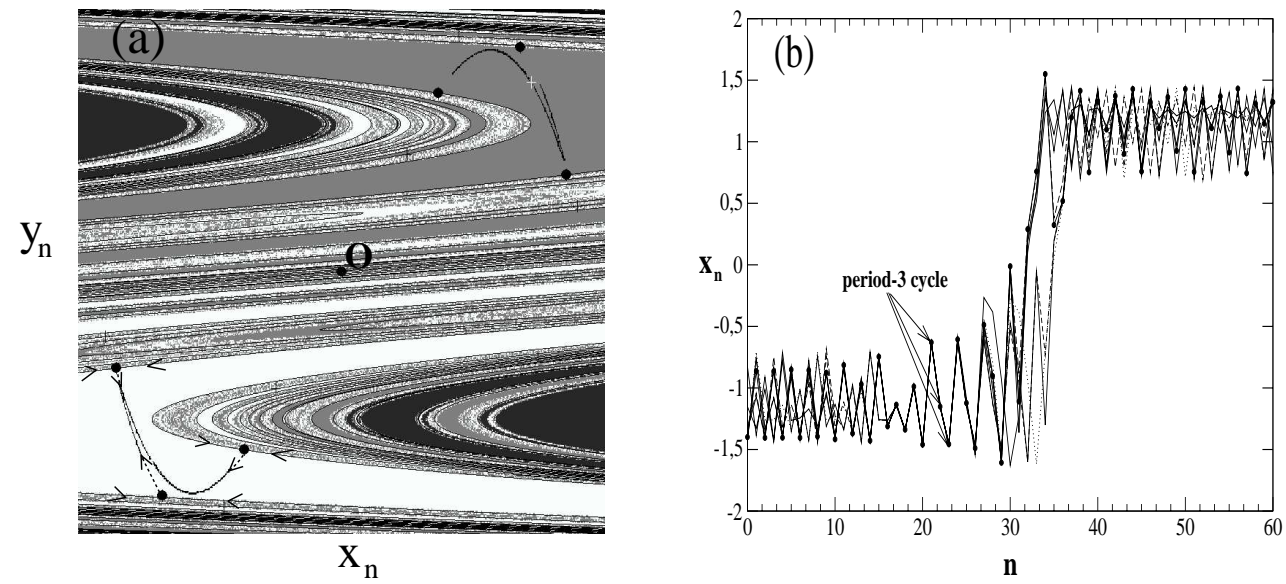

FIGURE 2. (a) Two co-existing chaotic attractors (full lines) in (2) at $a=2.7, b=0.2$ and their basins of attraction illustrated in grey and white respectively. The points of the period- 3 homoclinic saddles cycle are labeled by black dots, and the point $\mathrm{O}$ corresponds to the saddle at $(0,0)$; (b) Some typical escape trajectories collected with $D=10^{-5}$.

the basin of attraction the optimal force dies out. Note that no action is required to bring the system from $\mathrm{S} 1$ to the stable limit cycle.

Note that the saddle cycle of period 3 is not embedded in the NHA. It is probably the nearest saddle cycle to the boundary of the basin of attraction of the NHA in terms of the action variable, and can be considered as the boundary of the NHA itself.

\section{DISCRETE-TIME DYNAMICS}

As a second and very different example we consider fluctuational transitions between chaotic attractors separated by a fractal basin boundary in the two-dimensional map introduced my Holmes [3]:

$$
\begin{aligned}
& x_{i+1}=y_{i}, \\
& y_{i+1}=-b x_{i}+a y_{i}-y_{i}^{3}+\xi_{i},
\end{aligned}
$$

where $\xi_{i}$ is white Gaussian noise with $\left\langle\xi_{i}\right\rangle=0$ and $\left\langle\xi_{i}, \xi_{j}\right\rangle=2 D \delta_{i j}$. We choose (2) for our investigation because it exhibits a generic type of locally disconnected fractal basin boundary (see [10]), and because it reproduces most of the important features in the chaotic dynamics of the periodically driven Duffing oscillator [3]. The use of a discrete model brings the additional advantage of allowing us to speed up our numerical calculation for the case of low noise intensity.

We choose values of the control parameters $a$ and $b$ in (2) such that, in the purely deterministic case $(D=0)$, there are two co-existing chaotic attractors in phase space separated by a locally disconnected fractal basin boundary (see Fig. 2(a)). The fractal dimension of the boundary has been determined numerically as $d=1.8441$ by using the 


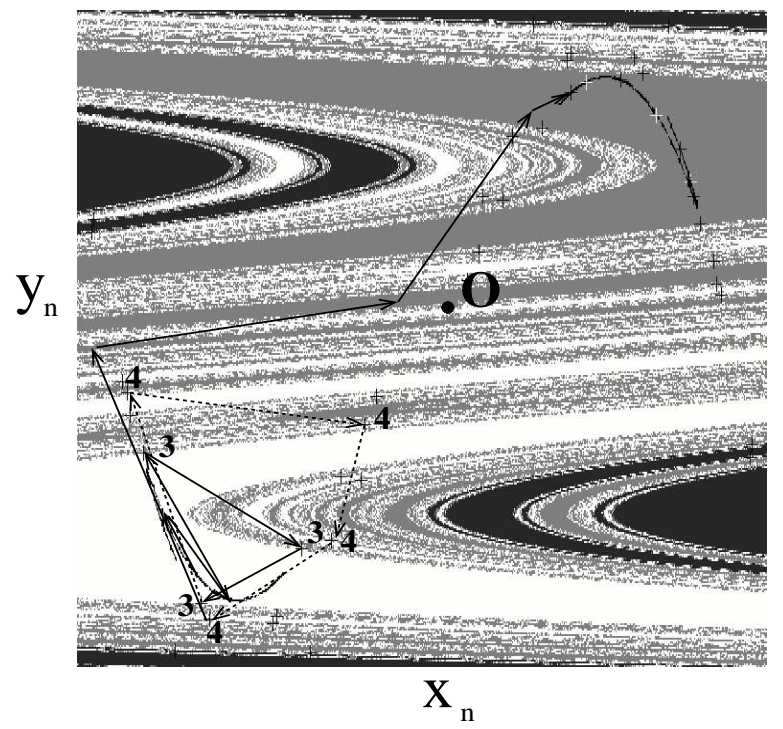

FIGURE 3. The escape trajectory in (2) from one chaotic attractor (full lines) to the other passes through the period-3 (full lines) and period-4 (dashed lines) original saddle cycles. The values of the control parameters and noise intensity are the same as in Fig. 1.

numerical "uncertainty exponent" technique introduced in [12]. We excite our system (2) with weak noise and collect trajectories that include escape paths from one chaotic attractor to the other (see Fig. 2(b)). The noise intensity was chosen in such a way that the mean escape time was essentially large; the characteristic relaxation time of an invariant measure on the corresponding chaotic set was estimated by us as $3 \times 10^{7}$ iterations. As can be clearly seen in Fig. 2(b), all escape trajectories have a part corresponding to the period-3 saddle cycle, apparently implying the presence of a boundary point located near, or directly on, the fractal boundary. Simple calculations have shown that the period3 saddle cycle does exist for the chosen parameter values and that it lies on the boundary. Moreover, its stable manifold (full line in Fig. 2(a)), lying in the boundary, detaches the open neighborhood including the chaotic attractor from the fractal basin boundary itself. One part of its unstable manifold belongs to the homoclinic structure forming the fractal boundary, whereas the other part (labeled by the dashed line in Fig. 2(a)) approaches the attractor. Thus, we can classify this period- 3 saddle as an accessible boundary point [13]. Indeed, by the definition given in [13] a boundary point $P$ is accessible from a given region if there is a curve of finite length connecting $P$ to an attractor in the interior of the region, such that no point of the curve lies in the boundary except for $P$. In our case, the part of the unstable manifold approaching the chaotic attractor plays the role of such a curve. Thus, in the present case, the period- 3 saddle point plays the role of the boundary condition.

It is well known that the global behavior of a chaotic dynamical system is in many respects determined by a homoclinic structure, i.e. by a set consisting of homoclinic saddle cycles resulting from tangencies of the stable and unstable manifolds of a saddle 


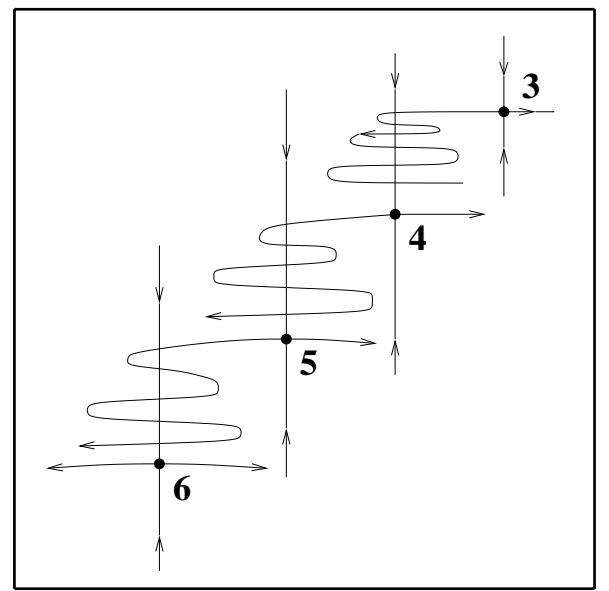

FIGURE 4. Heteroclinic crossings of the stable and unstable manifolds of different original saddles.

point. In our case, we observe an infinite sequence of saddle-node bifurcations of period $3,4,5,6,7 \ldots$, which occur at parameter values $d_{3}<d_{4}<d_{5}<d_{6}<d_{7} \ldots$ and are caused by the sequential tangencies of the stable and unstable manifolds of the saddle point $\mathrm{O}$ at $(0,0)$. The homoclinic orbits appearing as the result of these bifurcations were classified earlier as original saddles [13]. It was also shown that their stable and unstable manifolds cross each other in a hierarchical sequence: the unstable manifold of the period- 3 saddle crosses the stable manifold of the period- 4 saddle, the unstable manifold of the period- 4 saddle crosses the stable manifold of the period-5 saddle, etc. (see Fig. 4). Our numerical calculations have shown that these original saddles play a key role in the escape through a fractal basin boundary and that their hierarchy defines the structure of the escape paths. Fig. 3 shows a typical escape path obtained in numerical simulations. As clearly seen from this figure, a phase trajectory leaving the chaotic attractor penetrates into the fractal basin boundary through a small neighborhood of the period-3 saddle cycle, makes a few turns, and then approaches another period-4 original saddle point. After that it moves to the basin of the other chaotic attractor, reaching it in the next two or three iterations. In fact, the heteroclinic structure formed by numerous sequential crossings of the stable and unstable manifolds of original homoclinic saddles plays the role of the "staircase" allowing a trajectory to pass over the fractal basin boundary and defining the structure of the escape paths. Moreover, a hierarchical relation between original saddles can be revealed if we characterize them by a parameter $\mu$ equal to the ratio $\left|\lambda_{s}(P)\right| / \lambda_{u}(P)$ of the stable and unstable eigenvalues of the linearized deterministic flow at a saddle point $P$. Simple calculations show that, for the original saddles with periods $3,4,5,6,7,8 \ldots$ in (2), the following hierarchical sequence of index $\mu$ values occurs: $\mu_{3}=3.339, \mu_{4}=3.08, \mu_{5}=2.999, \mu_{6}=2.339, \mu_{7}=1.958, \mu_{8}=1.539$. The estimates of probabilities to find a fragment of the corresponding original saddle cycles in the escape trajectories are in a very good agreement with the hierarchical relation obtained above. By using the procedure described above for the continuous system, we 


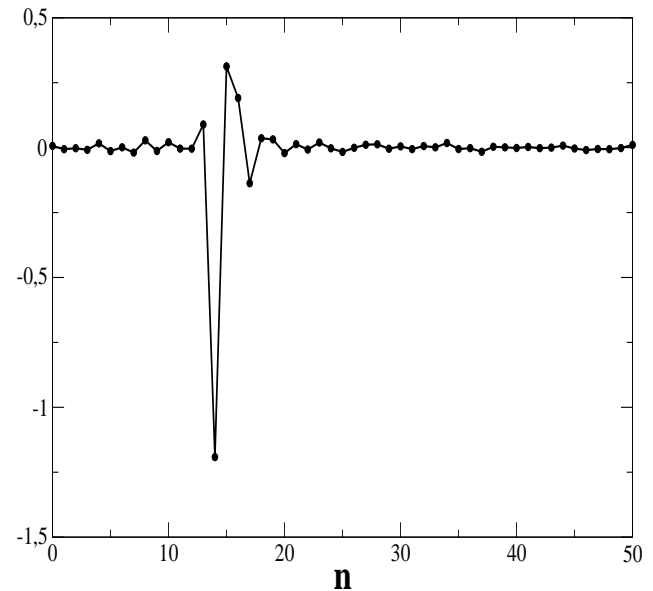

FIGURE 5. The optimal fluctuational force calculated at $a=2.7, b=0.2$ and $D=1.25 \cdot 10^{-5}$.

were able to calculate the optimal fluctuational force migrating the phase trajectory from one attractor to the other (see Fig. 5).

Thus we conclude that the control function and the corresponding boundary conditions can be found using the same technique [2] for both continuous and discrete time chaotic dynamics, and with both smooth and fractal basin boundaries around the chaotic attractor. Moreover the escape problem has in this case been reduced to the analysis of transitions between saddle cycles, in qualitative agreement with the well known statement that unstable cycles provide detailed invariant characterizations for dynamical systems of low intrinsic dimension.

The probability of escape from a chaotic attractor can now be calculated, therefore, as a product of the probabilities of transitions between saddle cycles. To date, the theoretical procedure for calculation of these probabilities remains an unsolved problem. It requires calculation of the invariant measure of the saddle cycles in the presence of noise, and the development of methods for calculation of the transition probability between cycles as a function of noise intensity. Although it is a difficult problem, the present work has brought a solution closer and will be applicable in many areas of physics, including e.g. the stability problem for ac-biased Josephson [1] junctions and the problem of fluctuational transitions in lasers [11].

\section{REFERENCES}

1. R. Kautz, Phys. Lett. A 125, 315-319 (1987).

2. I.A. Khovanov, D.G. Luchinsky, R. Mannella and P.V.E. McClintock, Phys. Rev. Lett. 85, 2100-2103 (2000).

3. P. Holmes, Phil. Trans. R. Soc. 292, 419-448 (1979).

4. A. L. Fradkov and A. Y. Pogromsky, Introduction to Control of Oscillations and Chaos, Vol. 35 of series on nonlinear science A (World Scientific, Singapore, 1998).

5. R. Zwanzig, J. Stat. Phys. 9, 215-220 (1973). 
6. M. I. Dykman and M. Krivoglaz, in Soviet Physics Reviews, edited by I.M. Khalatnikov (Harwood Academic Publishers, New York, 1984), Vol. 5, pp. 265-442.

7. M. I. Freidlin and A. D. Wentzel, Random Perturbations in Dynamical Systems (Springer, New-York, 1984).

8. L.C. Young, Lectures on the Calculus of Variations and Optimal Control Theory (Saunders, Philadelphia, 1969); P. Hagedorn, Non-linear Oscillations (Clarendon Press, Oxford, 1982).

9. M. I. Dykman et al, Phys. Rev. Lett. 68, 2718-2721 (1992).

10. S. V. McDonald, C. Grebogi, E. Ott, and J. A. Yorke, Physica 17D, 125-153 (1985).

11. V. N. Chizhevsky, E. V. Grigorieva, S. A. Kashchenko, Opt. Comm., 133, 189-195 (1997).

12. C. Grebogi, S. W. McDonald, E. Ott, and J.A. Yorke, Phys. Lett. 99A 415-418 (1983).

13. C. Grebogi, E. Ott, and J.A. Yorke, Physica 24D, 243-262 (1987). 
Copyright $\odot 2003$ EBSCO Publishing 
Copyright $\odot 2003$ EBSCO Publishing 
Copyright of AIP Conference Proceedings is the property of American Institute of Physics and its content may not be copied or emailed to multiple sites or posted to a listserv without the copyright holder's express written permission. However, users may print, download, or email articles for individual use. 